\title{
Bloqueo continuo del erector de la espina para dolor pélvico oncológico
}

\section{Continous block erector spine for pelvic oncologycal pain}

\author{
Ricardo Aguilar', Gabriel Carvajal², Andrés Rocha', Leslie Douglas', Andrea Ubertini
}

\begin{abstract}
Pain is a distressing symptom that affects $66 \%$ of cancer patients in advanced, metastatic or terminal disease. When systemic pharmacological analgesics fail to offer sufficient relief, invasive strategies such as continuous nerve blocks are often used for mid-term pain relief. Cancer related pelvic visceral and somatic pain is difficult to treat using continuous regional techniques. Standard approaches such as paravertebral block or chronic neuraxial techniques may be associated with potential severe complications, particularly in anticoagulated patients and patients with high infection risk. Erector spinae plane block (ESPB) has proven efficacious in anesthetic practice for visceral and somatic pain from several anatomical regions. This block provides somatic and visceral analgesia by local anesthetic diffusion into the neural foraminal, epidural and intercostal spaces and over several vertebral levels from a single non-neuraxial point of injection. We present the use of an ESPB with a catheter for the management of severe oncologic pain in a complex case with good results. We have found this block is helpful in a patient with Burkitt Lymphoma related pain for whom a temporary continuous block was needed before a definitive neuromodulation technique was offered. Current evidence for this technique to treat cancer pain is reviewed and we discuss possible areas of research.
\end{abstract}

\section{RESUMEN}

El dolor es un síntoma angustiante que afecta al $66 \%$ de los pacientes con cáncer en enfermedad avanzada, metastásica o terminal. Cuando la analgesia sistémica no ofrece un alivio suficiente, las estrategias mínimamente invasivas son una opción viable. El dolor visceral y somático pélvico relacionado con el cáncer es difícil de tratar utilizando técnicas regionales continuas. Los enfoques

\section{Key words:}

Chronic oncologycal pain,

spinal erector block

\section{Palabras clave:}

Dolor crónico

oncológico,

bloqueo erector de la espina

Unidad de Dolor, Centro Nacional de Rehabilitación. San José, Costa Rica.

Servicio de Medicina Paliativa, Departamento de Hemato-Oncología. Hospital México, Costa Rica.

Fecha de recepción: 03 de septiembre de 2019

Fecha de aceptación: 10 de octubre de 2019

\section{ORCID}

https://orcid.org/0000-0001-9647-1752

Correspondencia:

Dr. Andrés Rocha Romero

Email: rocharomeroandres@gmail.com 
estándar como el bloqueo paravertebral o las técnicas neuroaxiales crónicas pueden estar asociadas a posibles complicaciones graves, particularmente en pacientes anticoagulados y pacientes con alto riesgo de infección. El bloqueo del plano del erector de la espina ha demostrado su efectividad en la práctica anestésica en varias regiones anatómicas. Proporciona analgesia somática y visceral mediante difusión de anestésico local en los espacios foraminales, epidurales e intercostales en varios niveles vertebrales desde un único punto de inyección no neuroaxial. Presentamos el caso de uso de bloqueo ESP con un catéter para infusión de anestésicos en el tratamiento del dolor oncológico complejo logrando resultados óptimos. Hemos encontrado que esta técnica fue útil en un paciente con dolor relacionado con el linfoma de Burkitt para el que se necesitaba un bloqueo continuo temporal antes de que se ofreciera una técnica analgésica definitiva. Se revisa la evidencia actual de esta técnica para tratar el dolor por cáncer y discutimos áreas de investigación.

\section{Introducción}

I dolor es un síntoma angustiante común en pacientes con cáncer. Las tasas de prevalencia informadas son del $55 \%$ durante el tratamiento y $66 \%$ para enfermedad avanzada, metástasis o enfermedad terminal[1]. Las estrategias mínimamente invasivas como los bloqueos nerviosos continuos, a menudo se utilizan para el alivio del dolor a mediano plazo, cuando los tratamientos farmacológicos sistémicos no ofrecen una eficacia aceptable[2],[3]. Presentamos un caso complejo tratado con éxito con el bloqueo del plano erector de la espina (ESPB), una variante más de los bloqueos fasciales paraespinales que ha demostrado su eficacia en la práctica anestésica para el dolor de varias regiones anatómicas, aunque inicialmente fue descrito para la región torácica[4],[5].
Esta técnica proporciona analgesia somática y visceral posiblemente mediante la difusión de anestésico local en los espacios foraminales, epidurales e intercostales y en varios niveles vertebrales desde un único punto de inyección no neuroaxial[6],[7].

\section{Reporte de caso}

Presentamos el caso de una mujer de 23 años, 45 $\mathrm{kg}$ de peso, con diagnóstico de linfoma de Burkitt y dolor pélvico mixto somático y visceral severo (9/10 en una escala numérica de dolor) causado por una masa de $20 \times 17 \times 16 \mathrm{~cm}$ ubicada en su pelvis derecha (Figura 1 ). La lesión invadió el acetábulo derecho, la rama púbica y el sacro hasta el nivel S1, así como los tejidos blandos adyacentes y particularmente el cua-

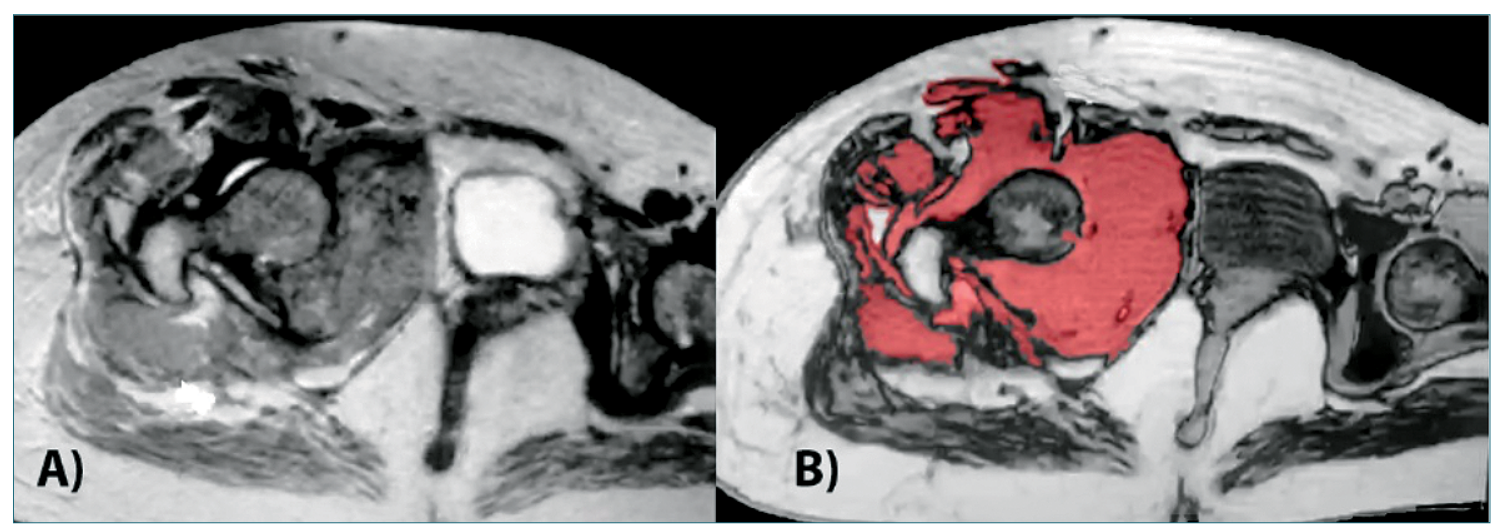

Figura 1. Resonancia magnética T1 de suelo pélvico. A) Tumor desplazando vejiga e invadiendo isquión y músculos paravertebrales; B) resaltado en color. 
drado lumbar, los músculos paravertebrales, el iliopsoas y el glúteo mayor. Debido a efectos adversos inaceptables con opioides sistémicos, se decidió pasar a manejo con catéter epidural tunelizado aplicando 30 mg diarios de morfina epidural, sin embargo, presentó signos clínicos de infección superficial; además, la paciente había tenido recientemente una trombosis venosa profunda y fue anticoagulada con enoxaparina $1 \mathrm{mg} / \mathrm{kg}$ dos veces al día por lo que el catéter se tuvo que retirar.

Se discutieron diferentes estrategias de manejo del dolor crónico entre la paciente, familiares y equipo médico siendo la opción más adecuada en ese momento un ESPB a nivel lumbar derecho. Se obtuvo el consentimiento informado por escrito y se procedió a realizar el mismo a nivel de L1 en sala de operaciones. Esto se realizó con una máquina de ultrasonido Logiq $E^{\circledR}(G E, E E$. UU.) y un transductor curvilíneo de 2-5,5 MHz colocado en un plano parasagital sobre la apófisis transversa del primer cuerpo vertebral lumbar derecho. Se insertó una aguja de $18 \mathrm{G} \times 100 \mathrm{~mm}$ entrando en plano, de cefálico a caudal al plano entre el músculo erector de la espina y la apofisis transversa derecha de L1 para administrar, previa aspiración, 20 $\mathrm{ml}$ de levobupivacaína $0,25 \%$ en bolos fraccionados de 5 en $5 \mathrm{ml}$.

Después del bolo, se colocó un catéter regional periférico 20G, se tunelizó y se aseguró con una película estéril transparente. Esto permitió un alivio del dolor casi completo durante las siguientes 12 horas (escala numérica del dolor 2/10). Se administraron bolos suplementarios de $20 \mathrm{ml}$ de la solución anestésica cada 12 horas. Este bloqueo fue efectivo para el dolor tanto visceral como somático y fue consistente con una distribución de los dermatomas de L1 a S2. Este régimen se mantuvo durante 15 días mientras la paciente completaba su quimioterapia, obteniendo un alivio del dolor adecuado durante este período. Posteriormente se colocó un sistema implantable de administración de fármacos intratecales para el tratamiento del dolor crónico por cáncer.

\section{Discusión}

Desde su primer reporte en 2016, el bloqueo ESPB solo se ha descrito para su uso continuo en el dolor por cáncer en 4 casos: carcinoma de vejiga, mesotelioma y el dolor relacionado con el cáncer de pulmón (Tabla 1). El presente caso ilustra la adaptación de este bloqueo en una paciente compleja como una estrategia puente hacia una técnica de dolor implantable[8], en una paciente que, además, cursaba con quimioterapia. Aún no hay ensayos aleatorios específicos del ESPB para el dolor crónico del cáncer, ni series retrospectivas con una tasa de inclusión significativa.

El dolor pélvico visceral y somático relacionado a cáncer es difícil de tratar utilizando técnicas regionales continuas en pacientes como la descrita. Los enfoques estándar como el bloqueo paravertebral o las técnicas neuroaxiales crónicas pueden estar asociados con posibles complicaciones graves, particularmente en pacientes anticoagulados[9] y pacientes con alto riesgo de infección[10]. Con el ESPB se ha comprobado alivio del dolor visceral y somático de varias regiones anatómicas, como cuello, tórax, abdomen, pelvis y extremidades inferiores[4],[11]-[17]. El bloqueo continuo ESPB puede ser una opción en pacientes con dolor relacionado con el cáncer visceral y somático que se plantea como puente hacia una técnica definitiva más compleja, que en nuestro caso fue una bomba analgésica intratecal.

Creemos que el uso de un catéter a nivel del ESP para el tratamiento del dolor oncológico severo en nuestra paciente tuvo buenos resultados y pudiera considerarse como una alternativa para el manejo de estos casos complejos. Las posibles áreas de investiga-

\begin{tabular}{|c|c|c|c|c|c|}
\hline Autor (año) & Sitio anatómico & Cáncer & Nivel bloqueado & $\begin{array}{l}\text { Duración } \\
\text { de catéter }\end{array}$ & Fármacos \\
\hline Forero $(2016)^{4}$ & Tórax costillas & $\begin{array}{l}\text { Carcinoma de ve- } \\
\text { jiga }\end{array}$ & T5 & 5 días & $\begin{array}{l}\text { Bupivacaína } 0,25 \%, 20 \mathrm{ml} \\
\text { diario }\end{array}$ \\
\hline $\operatorname{Ramos}(2018)^{18}$ & $\begin{array}{l}\text { Tórax irradiado a } \\
\text { hombro }\end{array}$ & $\begin{array}{l}\text { Mesotelioma pleu- } \\
\text { ral }\end{array}$ & T3 & 66 días & $\begin{array}{l}\text { Bupivacaína } 0,25 \%, 10 \mathrm{ml} \\
\text { c/8 h }\end{array}$ \\
\hline \multirow[t]{2}{*}{ Aydn $(2018)^{19}$} & $\begin{array}{l}\text { Tórax y lesiones } \\
\text { óseas }\end{array}$ & Cáncer de pulmón & $\mathrm{T} 2$ & 4 meses & $\begin{array}{l}10 \mathrm{~mL} \mathrm{0,25 \%} \text { bupivacaína } \\
\mathrm{c} / 8 \mathrm{~h}\end{array}$ \\
\hline & $\begin{array}{l}\text { Tórax, anterior y } \\
\text { posterior (T2 a T8) }\end{array}$ & Mesotelioma & T6 & & $\begin{array}{l}10 \mathrm{~mL} 0,25 \% \text { bupivacaína } 3 \\
\text { times a day }\end{array}$ \\
\hline
\end{tabular}


ción incluyen el impacto hemodinámico, las complicaciones del manejo de catéteres como desplazamiento, oclusión o infección en comparación con otras técnicas continuas de uso común, como la epidural. Se debe evaluar la eficacia y la seguridad a largo plazo y se deben estandarizar los regímenes de prescripción específicos. A nuestro entender este es el primer caso del uso del ESPB en un paciente con dolor por cáncer a nivel lumbar y de miembros inferiores recibiendo quimioterapia.

\section{Referencias}

1. van den Beuken-van Everdingen $\mathrm{MH}$, Hochstenbach LM, Joosten EA, Tjan-Heijnen VC, Janssen DJ. Update on Prevalence of Pain in Patients With Cancer: Systematic Review and MetaAnalysis. J Pain Symptom Manage. 2016 Jun;51(6):1070-1090. e9. https://doi.org/10.1016/j. jpainsymman.2015.12.340 PMID:27112310

2. Fuzier R, Izard P, Cabos C, Chaminade B, Pouymayou J. Chronic Cancer-Related Pain: Continuous Perineural Infusion of Local Anesthetics as Alternative to Systemic Analgesic Drugs. J Pain Palliat Care Pharmacother. 2016 Sep;30(3):195-200. https://doi. org/10.1080/15360288.2016.11 92082 PMID:27322898

3. Zinboonyahgoon N, Vlassakov K, Abrecht CR, Srinivasan S, Narang S. Brachial Plexus Block for Cancer-Related Pain: A Case Series. Pain Physician. 2015 Sep-Oct;18(5):E917-24. PMID:26431146

4. Forero M, Adhikary SD, Lopez H, Tsui C, Chin KJ. The Erector Spinae Plane Block: A Novel Analgesic Technique in Thoracic Neuropathic Pain. Reg Anesth Pain Med. 2016 Sep-Oct;41(5):621-7. https://doi.org/10.1097/ AAP.0000000000000451 PMID:27501016

5. Pfeiffer G, Oppitz N, Schöne S, Richter-Heine I, Höhne M, Koltermann C. Analgesie der Achselhöhle durch Para- vertebralkatheter in Laminatechnik. Anaesthesist. 2006 Apr;55(4):423-7. https://doi. org/10.1007/s00101-005-09690 PMID:16404582

6. Adhikary SD, Bernard S, Lopez $\mathrm{H}$, Chin KJ. Erector Spinae Plane Block Versus Retrolaminar Block: A Magnetic Resonance Imaging and Anatomical Study. Reg Anesth Pain Med. 2018 Oct;43(7):756-62. https://doi.org/10.1097/ AAP.0000000000000798 PMID:29794943

7. Schwartzmann A, Peng $P$, Maciel MA, Forero M. Mechanism of the erector spinae plane block: insights from a magnetic resonance imaging study. Can J Anaesth. 2018 Oct;65(10):1165-6. https:// doi.org/10.1007/s12630-0181187-y PMID:30076575

8. Smith TJ, Staats PS, Deer T, Stearns LJ, Rauck RL, BoortzMarx RL, et al.; Implantable Drug Delivery Systems Study Group. Randomized clinical trial of an implantable drug delivery system compared with comprehensive medical management for refractory cancer pain: impact on pain, drug-related toxicity, and survival. J Clin Oncol. 2002 Oct;20(19):4040-9. https://doi. org/10.1200/JCO.2002.02.118 PMID:12351602

9. Adhikary SD, Prasad A, Soleimani B, Chin KJ. Continuous Erector Spinae Plane Block as an Effective Analgesic Option in Anticoagulated Patients After Left Ventricular Assist
Device Implantation: A Case Series. J Cardiothorac Vasc Anesth. 2018;(April): https://doi. org/10.1053/j.jvca.2018.04.026 PMID:29753668

10. Bomberg $H$, Bayer I, Wagenpfeil S, Kessler P, Wulf H, Standl $\mathrm{T}$, et al. Prolonged Catheter Use and Infection in Regional Anesthesia: A Retrospective Registry Analysis. Anesthesiology. 2018 Apr;128(4):764-73. https://doi.org/10.1097/ ALN.0000000000002105 PMID:29420315

11. Hannig KE, Jessen C, Soni UK, Børglum J, Bendtsen TF. Erector Spinae Plane Block for Elective Laparoscopic Cholecystectomy in the Ambulatory Surgical Setting. Case Rep Anesthesiol. 2018 Apr;2018:5492527. https://doi. org/10.1155/2018/5492527 PMID:29805812

12. Krishna SN, Chauhan $S$, Bhoi D, Kaushal B, Hasija S, Sangdup $T$, et al. Bilateral Erector Spinae Plane Block for Acute Post-Surgical Pain in Adult Cardiac Surgical Patients: A Randomized Controlled Trial. J Cardiothorac Vasc Anesth. 2019 Feb;33(2):368-75. https://doi. org/10.1053/j.jvca.2018.05.050 PMID:30055991

13. Darling CE, Pun SY, Caruso TJ, Tsui BC. Successful directional thoracic erector spinae plane block after failed lumbar plexus block in hip joint and proximal femur surgery. J Clin Anesth. 2018 Sep;49:1-2. https://doi.org/10.1016/j. jclinane.2018.05.002 
PMID:29775780

14. Singh S, Chaudhary NK. Bilateral Ultasound Guided Erector Spinae Plane Block for Postoperative Pain Management in Lumbar Spine Surgery:

A Case Series. J Neurosurg Anesthesiol. 2018;(June): https://doi.org/10.1097/ ANA.0000000000000518 PMID:29965831

15. Aksu C, Gürkan Y. Opioid sparing effect of Erector Spinae Plane block for pediatric bilateral inguinal hernia surgeries. J Clin Anesth. 2018 Nov;50:62-3. https://doi.org/10.1016/j. jclinane.2018.06.048 PMID:29980003
16. Tulgar $S$, Kose HC, Selvi O, Senturk O, Thomas DT, Ermis MN, et al. Comparison of UltrasoundGuided Lumbar Erector Spinae Plane Block and Transmuscular Quadratus Lumborum Block for Postoperative Analgesia in Hip and Proximal Femur Surgery: A Prospective Randomized Feasibility Study. Anesth Essays Res. 2018 Oct-Dec;12(4):825-31. https://doi.org/10.4103/aer. AER_142_18 PMID:30662115

17. Tsui BC, Fonseca A, Munshey F, McFadyen G, Caruso TJ. The erector spinae plane (ESP) block: A pooled review of 242 cases. J Clin Anesth. 2019 Mar;53:2934. https://doi.org/10.1016/j. jclinane.2018.09.036

PMID:30292068

18. Ramos J, Peng P, Forero $M$. Long-term continuous erector spinae plane block for palliative pain control in a patient with pleural mesothelioma. Can J Anaesth. $2018 \mathrm{Jul}$;65(7):852-3. https://doi.org/10.1007/s12630018-1097-z PMID:29488178

19. Aydın T, Balaban O, Acar A. Ultrasound guided continuous erector spinae plane block for pain management in pulmonary malignancy. J Clin Anesth. 2018 May;46:63-4. https://doi.org/10.1016/j. jclinane.2018.01.023 PMID:29414621 